\title{
Predictors of Flare Following Etanercept Withdrawal in Patients with Rheumatoid Factor-negative Juvenile Idiopathic Arthritis Who Reached Remission while Taking Medication
}

\author{
Angela Aquilani, Denise Pires Marafon, Emiliano Marasco, Rebecca Nicolai, Virginia Messia, \\ Francesca Perfetti, Silvia Magni-Manzoni, and Fabrizio De Benedetti
}

\begin{abstract}
Objective. To evaluate the rate of flare after etanercept (ETN) withdrawal in patients with juvenile idiopathic arthritis (JIA) who attained clinical remission while taking medication, and to identify predictors of flare.

Methods. Patients were included with oligo- (oJIA) and rheumatoid factor-negative polyarticular JIA (pJIA) who received a first course of ETN for at least 18 months, maintained clinically inactive disease (CID) for at least 6 months during treatment, and were followed for 12 months after ETN withdrawal. Demographic and clinical features were collected at onset, at baseline (initiation of ETN), and at time of disease flare.

Results. After ETN withdrawal, 66 of the 110 patients enrolled (60\%) flared with arthritis (of whom 7 flared with concurrent anterior uveitis; none with uveitis alone). The median time to flare was 4.3 months (interquartile range 2.5-6.4) with no evident differences between oJIA and pJIA. The number and type of joints involved at baseline and characteristics of ETN treatment/discontinuation were not associated with flare. Patients who flared were more frequently males $(\mathrm{p}=0.034)$, positive for antinuclear antibody (ANA; $\mathrm{p}=0.047)$, and had higher values of $\mathrm{C}$-reactive protein $(\mathrm{CRP} ; \mathrm{p}=0.012)$ at baseline. These variables remained significantly associated with flare in a multivariate logistic analysis, a model accounting for only $14 \%$ of the variability of the occurrence of the flare.

Conclusion. Our results show that a significant proportion of patients with JIA who maintain CID for at least 6 months experience a relapse after ETN withdrawal. Male sex, presence of ANA, and elevated CRP at baseline were associated with higher risk of flare. (First Release May 1 2018; J Rheumatol 2018;45:956-61; doi:10.3899/jrheum.170794)
\end{abstract}

Key Indexing Terms:

JUVENILE IDIOPATHIC ARTHRITIS ETANERCEPT WITHDRAWAL REMISSION FLARE

Juvenile idiopathic arthritis (JIA) is the most common rheumatic condition in children and an important cause of short- and longterm disability. It represents a heterogeneous group of disorders characterized by childhood-onset chronic arthritis of unknown cause ${ }^{1}$.

From the Division of Rheumatology, Ospedale Pediatrico Bambino Gesù, Institute for Research and Health Care (IRCCS), Rome, Italy.

Supported by Pzifer Italia.

A. Aquilani, MD, Division of Rheumatology, Ospedale Pediatrico Bambino Gesù, IRCCS; D. Pires Marafon, MD, Division of Rheumatology,

Ospedale Pediatrico Bambino Gesù, IRCCS; E. Marasco, MD, Division of Rheumatology, Ospedale Pediatrico Bambino Gesù, IRCCS; R. Nicolai, $M D$, Division of Rheumatology, Ospedale Pediatrico Bambino Gesù, IRCCS; V. Messia, MD, Division of Rheumatology, Ospedale Pediatrico Bambino Gesù, IRCCS; F. Perfetti, MD, Division of Rheumatology, Ospedale Pediatrico Bambino Gesù, IRCCS; S. Magni-Manzoni, MD, Division of Rheumatology, Ospedale Pediatrico Bambino Gesù, IRCCS; F. De Benedetti, MD, PhD, Division of Rheumatology, Ospedale Pediatrico Bambino Gesù, IRCCS.

Address correspondence to Dr. A. Aquilani, Division of Rheumatology, Ospedale Pediatrico Bambino Gesù, IRCCS, Piazza Sant'Onofrio 4, 00165 Rome, Italy.E-mail: angela.aquilani@gmail.com

Accepted for publication February 6, 2018.
The current classification of JIA is a subject for debate ${ }^{2}$. For example, antinuclear antibody (ANA) presence has been suggested as a descriptor to identify a homogeneous group of JIA patients with common clinical features ${ }^{3}$. Nonetheless, the classification proposed by the International League of Associations for Rheumatology (ILAR) remains the reference. According to the ILAR classification, JIA is classified into 7 different categories of arthritis based on the number of joints involved, serologic findings, and extraarticular manifestations ${ }^{4}$. Currently the treatment of children with JIA includes nonsteroidal antiinflammatory drugs, glucocorticoids, and disease-modifying antirheumatic drugs (DMARD). Methotrexate (MTX) is the first-line DMARD most commonly used. However, MTX is effective only in a proportion of patients ${ }^{5}$. Tumor necrosis factor inhibitors (TNFi) have been shown to be effective in JIA patients with an unsatisfactory response to MTX. Etanercept (ETN) is the first TNFi approved for the treatment of polyarticular course JIA. Since the publication of the first randomized controlled trial of ETN in JIA ${ }^{6}$, the number of children treated with ETN

Personal non-commercial use only. The Journal of Rheumatology Copyright $\odot$ 2018. All rights reserved 
has increased rapidly. A large number of patients with JIA treated with ETN attain long-lasting remission. While some clinical variables have been found to be associated with response to $\mathrm{ETN}^{7,8}$, little is known about the risk of flare after ETN withdrawal. Five previous studies have addressed the issue, but definitive conclusions could not be drawn because of the small number of patients, the heterogeneity in the TNFi analyzed, the variability of the patient populations studied, and the different treatment protocols used (both duration of

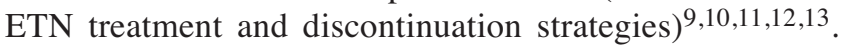
Indeed, no guideline exists to recommend appropriate withdrawal of TNFi once remission status has been achieved. Further, treatment practice on the discontinuation of ETN varies among different centers and remains provider-dependent.

However, although ETN withdrawal is associated with a flare risk, this must be balanced against the risk (in part unknown) of unnecessarily prolonging treatment with a selective cytokine inhibitor in a patient who would maintain a disease-free status even without treatment.

The aim of our current study was to evaluate the rate of disease flare during the first 12 months following ETN withdrawal in patients with oligoarticular (OJIA) and polyarticular JIA (pJIA) who have attained remission while taking the medication. A second aim was to evaluate whether standard clinical and demographic features allow identification of possible predictors of flare.

\section{MATERIALS AND METHODS}

We conducted a retrospective chart review of patients with JIA treated in our center with ETN between May 2005 and June 2016 who discontinued ETN because of persistent clinically inactive disease (CID) while taking medication. Inactive disease was defined according to Wallace criteria ${ }^{14}$.

Patients were included if they received first course of ETN for at least 18 months (once weekly at $0.8-1 \mathrm{mg} / \mathrm{kg} /$ dose, up to $50 \mathrm{mg}$ ), maintained CID for at least 6 months while receiving ETN therapy (i.e., remission on medication), and were followed up to 12 months after ETN withdrawal. To study a more homogeneous group, we enrolled in the study only patients with oJIA, including persistent oJIA and extended oJIA, or pJIA rheumatoid factor (RF)-negative. Patients were excluded if they had systemic JIA, were pJIA RF-positive, had enthesitis-related arthritis, or unclassified JIA according to the ILAR classification criteria ${ }^{4}$. In addition, patients were excluded if they were previously exposed to other biologics, in therapy with medications other than MTX and ETN during the 3 months prior to the withdrawal of ETN, noncompliant to therapy, or if they had limited followup or incomplete medical records. In the absence of recommendations, modalities for ETN withdrawal were not standard: ETN withdrawal was performed abruptly (i.e., suspension at a single visit) or gradually (i.e., dose reduced or interval of 2 consecutive injections increased, for any time of tapering).

For each patient, we recorded the demographic and clinical features at onset, including JIA ILAR subtype and ANA positivity (at least 2 positive results on indirect immunofluorescence at a titer of $\geq 1: 160,3$ mos apart in the first months of the disease $)^{15}$, and the following variables at ETN start (baseline): disease duration, type of active joints, number of active joints, erythrocyte sedimentation rate (ESR), C-reactive protein (CRP), and concomitant treatment with MTX. The following items were collected at time of flare or 12 months after ETN withdrawal: duration of ETN treatment, time to CID on ETN, duration of CID while receiving and not receiving medication, concomitant medications with MTX at ETN withdrawal, occur- rence of flare, time to flare from ETN withdrawal, and the type of joints in which flare occurred. Flare was defined by the occurrence of active arthritis or uveitis after ETN withdrawal. A CRP level $>0.5 \mathrm{mg} / \mathrm{dl}$ and ESR value $>20 \mathrm{~mm} / \mathrm{h}$ were considered elevated. Further, patients were grouped according to the presence or absence of ANA. Baseline and followup features were compared between the patients who experienced a flare and those who did not. The study protocol was approved by the Ethical Committee of the Children's Hospital Bambino Gesù (Protocol number 601.12).

Statistical analysis. Quantitative data were presented as median and interquartile range (IQR), while categorical data as absolute numbers and percentages. Proportions were compared using Fisher's exact test. Comparisons between groups were performed using the Mann-Whitney $\mathrm{U}$ test. A p value $<0.05$ was considered significant. Statistical analysis was performed using the Graph Pad Prism 5 software. Multiple linear regression analysis was used to explore the relationship of occurrence of flare (dependent variable) during the first 12 months after ETN withdrawal using the following predictor variables: sex, presence of ANA, and elevated CRP at baseline.

\section{RESULTS}

Study population. A total of 110 patients with oJIA (24 persistent oJIA and 33 extended oJIA) or pJIA who received first course of ETN for least 18 months and discontinued treatment owing to the achievement of CID were followed during the study period. The median age at disease onset was 3.6 years (IQR 1.8-8.5) and 70\% of the patients were female. When patients were divided according to presence or absence of ANA, ANA-positive patients had a younger age at onset and were more frequently oJIA, as expected (Table 1).

Baseline disease features at ETN start, treatment duration, time to CID, and time from CID to ETN withdrawal are shown in Table 2. ETN was started after a median disease duration of 9.1 months. Because ETN treatment is generally prescribed in patients with extended oJIA (i.e., extension of arthritis to $>4$ joints after the first 6 mos of disease) and/or in patients with fewer active joints and resistant to repeated intraarticular injections and DMARD, disease duration at ETN start was significantly longer in patients with oJIA (median 15.2 mos) compared to patients with pJIA (5.1 mos). As expected, the number of active joints at baseline was higher in patients with pJIA. However, no differences were found in CRP levels and ESR values between the groups. The median duration of treatment was 22.2 months (IQR 18.4-27.9), with no differences among the groups. In the group of responders to ETN, CID was quickly attained after a median treatment duration of 4.5 months (IQR 2.2-7.3). It is worth noting that ANA-positive patients attained CID before ANA-negative patients (median time to CID $4.3 \mathrm{mos}$ vs 6.3 mos, $p=0.016$ ). After attaining CID, the median duration of ETN treatment before withdrawal was 14.6 months (IQR 11.1-21.3), with no significant differences between the groups.

Frequency of flare and features associated with flares after withdrawal of ETN. In the 12 months of followup after ETN withdrawal, a total of 66 of the 110 patients $(60 \%)$ flared with

Personal non-commercial use only. The Journal of Rheumatology Copyright $\odot$ 2018. All rights reserved 
Table 1. Demographics and clinical characteristics of patients with oJIA and pJIA included in the study.

\begin{tabular}{|c|c|c|c|c|c|c|c|}
\hline Characteristics & Total, $\mathrm{n}=110$ & oJIA, $\mathrm{n}=57$ & pJIA, $\mathrm{n}=53$ & $\mathrm{p}$ & $\mathrm{ANA}+, \mathrm{n}=71$ & ANA-, $\mathrm{n}=39$ & $\mathrm{p}$ \\
\hline Sex, female ${ }^{1}$ & $77(70)$ & $42(73.7)$ & $35(66)$ & 0.41 & $49(69)$ & $28(71.8)$ & 0.83 \\
\hline Age at disease onset, $\mathrm{yrs}^{2}$ & $3.6(1.8-8.5)$ & $3.0(1.8-6.1)$ & $5.0(1.9-9.6)$ & 0.13 & $2.5(1.6-5.1)$ & $8.0(3.5-9.9)$ & $<0.001$ \\
\hline No. active joints at onset ${ }^{2}$ & $3.5(1.0-7.0)$ & $1(1-2)$ & $7.0(6.0-11.5)$ & $<0.001$ & $2(1-6)$ & $5(2-10)$ & 0.01 \\
\hline
\end{tabular}

${ }^{1} \mathrm{~N}(\%)$, Fisher's exact test. ${ }^{2}$ Median (IQR), Mann-Whitney U test. oJIA: oligoarticular juvenile idiopathic arthritis; pJIA: polyarticular JIA; ANA: antinuclear antibody; IQR: interquartile range.

Table 2. Clinical and laboratory features of patients with JIA included in the study at baseline (i.e., initiation of ETN treatment). Data are median (IQR), Mann-Whitney U test.

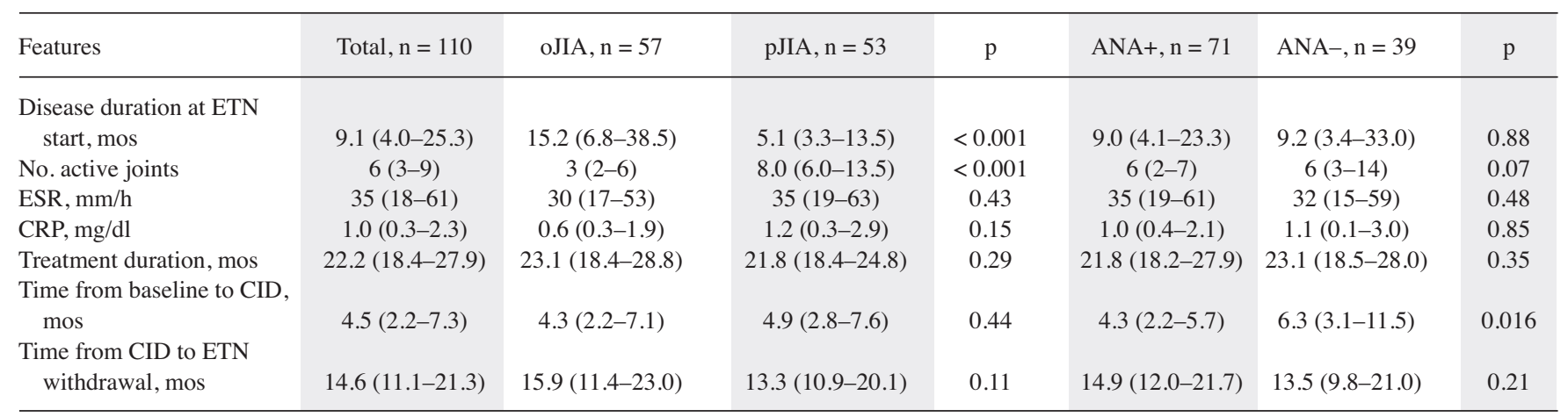

JIA: juvenile idiopathic arthritis; ETN: etanercept; IQR: interquartile range; oJIA: oligoarticular JIA; pJIA: polyarticular JIA; ANA: antinuclear antibody; ESR: erythrocyte sedimentation rate; CRP: C-reactive protein; CID: clinically inactive disease.

active arthritis, while $44(40 \%)$ did not present reactivation of arthritis. Seven of 66 patients (10.6\%) flared with arthritis and concurrent anterior uveitis; 5 of them had presented iridocyclitis before ETN treatment, and for 2 it was the first episode. None flared with anterior uveitis alone. The median time to flare was 4.3 months (IQR 2.5-6.4), with no evident differences between the groups. About one-third of the flares were late flares, occurring from month 7 to month 12 . As expected, patients with pJIA flared with a higher number of active joints (Table 3 ).

We then wanted to identify potential variables that could predict the probability of a flare following ETN withdrawal after attaining remission while taking medication. Table 4 shows the comparisons of demographic characteristics, clinical and laboratory features at baseline, concomitant MTX treatments, and characteristics of ETN treatment and discontinuation.

Patients who flared were more frequently men, more frequently ANA-positive, and had higher values of CRP at baseline than patients who did not flare. The rate of flare was not associated with the pJIA or oJIA subtype at onset, nor with having persistent oJIA or extended oJIA (data not shown). The number and type of joints with active arthritis at baseline was not associated with flare. Baseline or concomitant treatment with MTX at ETN withdrawal was not associated with flare. Regarding treatment with ETN, the

Table 3. Flare occurrence and flare features in the 66 patients who experienced a flare $<12$ months after the start of ETN therapy. Data are n (\%), using Fisher's exact test, or median (IQR), using Mann-Whitney U test.

\begin{tabular}{|c|c|c|c|c|c|c|c|}
\hline Flare Features & Total, $\mathrm{n}=66$ & oJIA, $n=33$ & pJIA, $n=33$ & $\mathrm{p}$ & $\mathrm{ANA}+, \mathrm{n}=48$ & ANA-, $n=18$ & $\mathrm{p}$ \\
\hline \multicolumn{8}{|l|}{ Flare 0-6 mos from } \\
\hline ETN withdrawal & $45(68.2)$ & $21(63.6)$ & $24(72.7)$ & 0.60 & $33(68.8)$ & $12(66.7)$ & 1.0 \\
\hline ETN withdrawal & $21(31.8)$ & $12(36.4)$ & $9(27.3)$ & & $15(31.3)$ & $6(33.3)$ & \\
\hline $\begin{array}{l}\text { Time from ETN withdrawal to } \\
\text { flare, mos }\end{array}$ & $4.3(2.5-6.4)$ & $4.0(2.6-7.6)$ & $4.4(2.4-6.1)$ & 0.78 & $4.1(2.4-6.8)$ & $4.7(2.7-6.4)$ & 0.64 \\
\hline
\end{tabular}

ETN: etanercept; IQR: interquartile range; oJIA: oligoarticular juvenile idiopathic arthritis; pJIA: polyarticular JIA; ANA: antinuclear antibody. 
Table 4. Demographic characteristics, clinical and laboratory features at baseline, concomitant MTX treatments, and characteristics of ETN treatment and discontinuation in JIA patients who flare and those who did not flare in the 12 months of followup after ETN withdrawal, after attaining remission. Data are $n$ (\%) calculated by Fisher's exact test, or median (IQR) using the Mann-Whitney U test.

\begin{tabular}{|c|c|c|c|c|}
\hline Characteristics & All Patients, $n=110$ & Flare, $n=66$ & No Flare, $n=44$ & $\mathrm{p}$ \\
\hline Sex, female & $77(70)$ & $41(62.1)$ & $36(81.8)$ & 0.034 \\
\hline Sex, male & $33(30)$ & $25(37.9)$ & $8(18.2)$ & \\
\hline Age at disease onset, yrs & $3.6(1.8-8.5)$ & $3.7(2.0-8.7)$ & $3.1(1.7-8.4)$ & 0.60 \\
\hline Disease duration at diagnosis. mos & $2.3(1.5-4.1)$ & $2.3(1.5-3.3)$ & $2.5(1.5-5.0)$ & 0.44 \\
\hline oJIA & $57(51.8)$ & $33(50)$ & $24(54.5)$ & 0.70 \\
\hline pJIA & $53(48.2)$ & $33(50)$ & $20(45.5)$ & \\
\hline ANA-positive ( $\geq 1: 160)$ & $71(64.5)$ & $48(72.7)$ & $23(52.3)$ & 0.047 \\
\hline ANA-negative & $39(35.5)$ & $18(27.3)$ & $21(47.3)$ & \\
\hline CRP at baseline, $\mathrm{mg} / \mathrm{dl}$ & $1.0(0.3-2.3)$ & $1.2(0.4-3.2)$ & $0.4(0.2-1.5)$ & 0.012 \\
\hline No. patients with CRP $>$ ULN & $66(60)$ & $46(69.7)$ & $20(45.5)$ & 0.017 \\
\hline No. active joints at baseline & $6(3-9)$ & $5.0(3.0-7.3)$ & $6.0(3.0-10.5)$ & 0.40 \\
\hline \multicolumn{5}{|l|}{ Joint involved at baseline } \\
\hline Cervical spine & $10(9.1)$ & $6(9.1)$ & $4(9.1)$ & 1.0 \\
\hline Temporomandibular & $10(9.1)$ & $5(7.6)$ & $5(11.4)$ & 0.52 \\
\hline Elbow & $25(22.7)$ & $18(27.3)$ & $7(15.9)$ & 0.25 \\
\hline Wrist & $39(35.5)$ & $21(31.8)$ & $18(40.9)$ & 0.42 \\
\hline Metacarpophalangeal & $10(9.1)$ & $4(6.1)$ & $6(13.6)$ & 0.19 \\
\hline Proximal interphalangeal & $59(53.6)$ & $34(51.5)$ & $25(56.8)$ & 0.70 \\
\hline Hip & $15(13.6)$ & $6(9.1)$ & $9(20.5)$ & 0.10 \\
\hline Knee & $82(74.5)$ & $48(72.7)$ & $34(77.3)$ & 0.66 \\
\hline Ankle & $79(71.8)$ & $50(75.8)$ & $29(65.9)$ & 0.29 \\
\hline Disease duration at baseline, $\operatorname{mos}$ & $9.1(4.0-25.3)$ & $8.1(3.7-21.0)$ & $9.7(5.3-39.4)$ & 0.07 \\
\hline Concomitant MTX at baseline & $106(96.4)$ & $63(95.5)$ & $43(97.7)$ & 0.65 \\
\hline Concomitant MTX at ETN stop & $87(79.1)$ & $50(75.8)$ & $37(84.1)$ & 0.34 \\
\hline Time from ETN start to CID, mos & $4.5(2.2-7.3)$ & $4.8(2.2-6.8)$ & $4.2(2.9-9.3)$ & 0.61 \\
\hline Time from CID to ETN withdrawal, mos & $14.6(11.1-21.3)$ & $14.6(11.9-21.9)$ & $14.7(10.4-20.7)$ & 0.40 \\
\hline ETN treatment duration & $22.2(18.4-27.9)$ & $22.2(18.4-28.2)$ & $21.9(18.5-27.1)$ & 0.85 \\
\hline ETN abrupt withdrawal & $82(74.5)$ & $52(78.8)$ & $30(68.2)$ & 0.27 \\
\hline ETN gradual reduction & $28(25.5)$ & $14(21.2)$ & $14(31.8)$ & \\
\hline
\end{tabular}

ANA: antinuclear antibody; ULN: upper limit of normal; CRP: C-reactive protein; MTX: methotrexate; ETN: etanercept; JIA: juvenile idiopathic arthritis; IQR: interquartile range; CID: clinically inactive disease; oJIA: oligoarticular JIA; pJIA: polyarticular JIA.

duration of CID during treatment before ETN withdrawal, ETN treatment duration, or modality of ETN withdrawal were not associated with flare.

A logistic regression analysis was performed with occurrence of flare in the 12-month followup period after ETN withdrawal as the dependent variable. The following predictors were analyzed: sex, presence of ANA, elevated CRP at baseline, and disease duration according to association with flare, in univariate analysis. In this logistic multivariate analysis, male sex, presence of ANA, and elevated $\mathrm{CRP}$ at baseline remained significant predictors of flare ( $p=0.020, p=0.047$, and $p=0.012$, respectively), confirming the results obtained in the univariate analysis. The model based on these variables accounted for $13.9 \%$ of the variability of the occurrence of the flare during the first year after ETN discontinuation.

\section{DISCUSSION}

In this retrospective monocentric study, we found that following ETN withdrawal after attaining remission while taking medication, $60 \%$ of the patients with oJIA or pJIA presented a disease flare within 12 months. Male sex, ANA positivity, and abnormal CRP at baseline were associated with moderately increased risk of flare.

The percent of flare that we observed is similar to that reported in the available studies. It should be noted that all the studies except 1 that reported on disease flare after ETN withdrawal included a small number of patients ranging from 11 to 39 , with an overall flare rate of $60-70 \%$ in the first 12 months after ETN withdrawal. In the large study conducted by Baszis, et al of 136 patients who discontinued treatment after remission, $68 \%$ experienced a disease flare within 12 months of the withdrawal of a $\mathrm{TNFi}^{12}$. In all studies, including ours, the frequency of flare was slightly higher in the first 6 months after ETN withdrawal ${ }^{9,10,11,13}$.

It should be mentioned that these studies (except for the study by Pratsidou-Gertsi, et al) enrolled patients with all subsets of JIA, resulting in rather heterogeneous groups, with oJIA and pJIA representing about two-thirds of the patients and one-third encompassing all the other JIA subtypes ${ }^{9,10,11,12,13}$. In addition, duration of ETN treatment varied widely from a few months to several years. Moreover,

Personal non-commercial use only. The Journal of Rheumatology Copyright @ 2018 . All rights reserved. 
the duration of CID before ETN withdrawal was quite variable, ranging from 1 month to some years. In these studies, possibly because of the small numbers, the heterogeneity of the patients included, and the variability of the treatment duration and CID period before withdrawal, no indicator for successful discontinuation of ETN was identified ${ }^{9,10,11,12,13}$. In an attempt to analyze a more homogeneous group, we chose to include only patients with oJIA and pJIA. Further, a minimal treatment duration of 18 months was required. To avoid the confusing factor of a too-early ETN discontinuation, we included only patients in which ETN was withdrawn after at least 6 months of CID (i.e., remission while taking medication, according to Wallace criteria ${ }^{14}$ ).

To identify potential predictors of flare after ETN withdrawal, we investigated the association of flare following ETN withdrawal with duration of CID before withdrawal and modality of ETN withdrawal (abrupt vs gradual). Indeed, in the study by Prince, et al of 19 patients (of whom 7 had pJIA and 3 oJIA), longer CID while taking medication and gradual ETN withdrawal were associated with longer clinical remission after discontinuation ${ }^{9}$. In a recent work, it was reported that patients in all subsets of JIA who received biologics (including ETN, adalimumab, infliximab, anakinra, rituximab, and abatacept) more than 2 years after achieving CID had a higher probability of maintaining such remission while not receiving therapy ${ }^{15}$. Among the patients included in our study, only 7 had CID > 2 years before ETN withdrawal, and 6/7 flared following ETN withdrawal. Therefore, in our study, in a more homogeneous group of patients, neither longer time of CID before ETN withdrawal nor gradual ETN withdrawal were associated with reduced risk of flare. In particular, regarding gradual versus abrupt withdrawal in JIA, similar findings are reported in the study by Remesal, et al on 26 patients ${ }^{10}$, while the other studies, including the larger study by Baszis, et al, did not evaluate this variable $9,11,12,13$. Data in adult RA support the conclusion that gradual discontinuation may yield a better control of flare. Indeed, in a randomized clinical trial in adults with rheumatoid arthritis in which patients taking MTX and ETN who achieved clinical remission (28-joint count Disease Activity Score <2.6) were randomized to receive a reduced dose of ETN plus MTX or MTX alone, the proportion of patients who maintained remission was higher in patients randomized to ETN at a reduced dose compared to patients randomized to MTX alone ${ }^{16}$. In our study, patients were not randomized, only $25 \%$ had a gradual (i.e., not abrupt) discontinuation, and the length of time each patient spent on an every-other-week regimen was variable. A controlled study is necessary to address the potential advantage of gradual ETN discontinuation in JIA.

We found that male sex, ANA positivity, and elevated CRP at baseline were associated with flare after ETN discontinuation. ANA positivity has been reported to be associated with flare in a study that enrolled patients with all JIA forms and included all biologics ${ }^{17}$, but not in a more recent report by Simonini, et $a l^{15}$. In our study, these variables remained significantly associated with flare in a multivariate logistic analysis. However, this model accounted only for $14 \%$ of the variability of the occurrence of the flare after ETN discontinuation. Nevertheless, albeit significantly associated with flare, we conclude that male sex, ANA positivity, and elevated CRP are variables of little relevance in clinical practice for guiding therapeutic decisions.

Our results, even in a rather homogeneous group of patients and with standardized treatment duration and time on CID before ETN discontinuation, show that basic clinical and demographic variables do not allow correct identification of patients who will flare after ETN discontinuation. Other predictors are needed. The predictive value of serum MRP8/14 levels in identifying relapse after discontinuation of therapy with MTX or ETN in patients who maintained clinical remission was evaluated in previous studies ${ }^{18,19}$. Although higher levels of the myeloid-related proteins MRP8/14 are associated with higher risk of flare, the positive and negative predictive values are not sufficiently high to guide clinical decision making. We have recently observed that a low rate of increase in peripheral blood switched memory B cells is associated with absence of disease flare during ETN treatment ${ }^{20}$. Musculoskeletal ultrasound detection of subclinical synovitis in patients with clinically defined inactive disease has been suggested as a useful tool to identify residual inflammation ${ }^{21}$, though the issue is being debated and investigated ${ }^{18,19}$. Indeed, in the only available prospective, albeit small, study, ultrasound-detected synovial abnormalities alone did not predict an early flare of synovitis in the affected joints ${ }^{22}$. Therefore, large studies in a multicenter setting aimed at investigating multidimensional indicators are needed, possibly including clinical variables, blood biomarkers of residual inflammation, and imaging studies.

\section{REFERENCES}

1. Ravelli A, Martini A. Juvenile idiopathic arthritis. Lancet 2007;369:767-78.

2. Martini A. It is time to rethink juvenile idiopathic arthritis classification and nomenclature. Ann Rheum Dis 2012;71:1437-9.

3. Ravelli A, Varnier GC, Oliveira S, Castell E, Arguedas O, Magnani A, et al. Antinuclear antibody-positive patients should be grouped as a separate category in the classification of juvenile idiopathic arthritis. Arthritis Rheum 2011;63:267-75.

4. Petty RE, Southwood TR, Manners P, Baum J, Glass DN, Goldenberg J, et al. International League of Associations for Rheumatology classification of juvenile idiopathic arthritis: second revision, Edmonton, 2001. J Rheumatol 2004;31:390-2.

5. Stoll ML, Cron RQ. Treatment of juvenile idiopathic arthritis in the biologic age. Rheum Dis Clin North Am 2013;39:751-66.

6. Lovell DJ, Giannini EH, Reiff A, Cawkwell GD, Silverman ED, Nocton JJ, et al. Etanercept in children with polyarticular juvenile rheumatoid arthritis. Pediatric Rheumatology Collaborative Study Group. N Engl J Med 2000;342:763-9.

Personal non-commercial use only. The Journal of Rheumatology Copyright (C) 2018. All rights reserved 
7. Otten MH, Prince FH, Armbrust W, ten Cate R, Hoppenreijs EP, Twilt M, et al. Factors associated with treatment response to etanercept in juvenile idiopathic arthritis. JAMA 2011;306:2340-7.

8. Solari N, Palmisani E, Consolaro A, Pistorio A, Viola S, Buoncompagni A, et al. Factors associated with achievement of inactive disease in children with juvenile idiopathic arthritis treated with etanercept. J Rheumatol 2013;40:192-200.

9. Prince FH, Twilt M, Simon SC, van Rossum MA, Armbrust W, Hoppenreijs EP, et al. When and how to stop etanercept after successful treatment of patients with juvenile idiopathic arthritis. Ann Rheum Dis 2009;68:1228-9.

10. Remesal A, De Inocencio J, Merino R, Garcia-Consuegra J. Discontinuation of etanercept after successful treatment in patients with juvenile idiopathic arthritis. J Rheumatol 2010;37:1970-1.

11. Pratsidou-Gertsi P, Trachana M, Pardalos G, Kanakoudi-Tsakalidou F. A follow-up study of patients with juvenile idiopathic arthritis who discontinued etanercept due to disease remission. Clin Exp Rheumatol 2010;28:919-22.

12. Baszis K, Garbutt J, Toib D, Mao J, King A, White A, et al. Clinical outcomes after withdrawal of anti-tumor necrosis factor alpha therapy in patients with juvenile idiopathic arthritis: a twelve-year experience. Arthritis Rheum 2011;63:3163-8.

13. Postepski J, Kobusinska K, Olesinska E, Osinska V, OpokaWiniarska V. Clinical remission in juvenile idiopathic arthritis after termination of etanercept. Rheumatol Int 2013;33:2657-60.

14. Wallace CA, Ruperto N, Giannini E. Preliminary criteria for clinical remission for select categories of juvenile idiopathic arthritis. J Rheumatol 2004;31:2290-4.

15. Simonini G, Ferrara G, Pontikaki I, Scoccimarro E, Giani T, Taddio
A, et al. Flares after withdrawal of biologic therapies in juvenile idiopathic arthritis: Clinical and laboratory correlates of remission duration. Arthritis Care Res 2017 Oct 3 (E-pub ahead of print).

16. Emery P, Hammoudeh M, FitzGerald O, Combe B, Martin-Mola E, Buch $\mathrm{MH}$, et al. Sustained remission with etanercept tapering in early rheumatoid arthritis. N Engl J Med 2014;371:1781-92.

17. Guzman J, Oen K, Huber AM, Watanabe Duffy K, Boire G, Shiff N, et al. The risk and nature of flares in juvenile idiopathic arthritis: results from the ReACCh-Out cohort. Ann Rheum Dis 2016;75:1092-8.

18. Foell D, Wulffraat N, Wedderburn LR, Wittkowski H, Frosch M, Gerss J, et al. Methotrexate withdrawal at 6 vs 12 months in juvenile idiopathic arthritis in remission: a randomized clinical trial. JAMA 2010;303:1266-73.

19. Anink J, Van Suijlekom-Smit LW, Otten MH, Prince FH, van Rossum MA, Dolman KM, et al. MRP8/14 serum levels as a predictor of response to starting and stopping anti-TNF treatment in juvenile idiopathic arthritis. Arthritis Res Ther 2015;17:200.

20. Marasco E, Aquilani A, Cascioli S, Moneta GM, Caiello I, Farroni C, et al. Switched memory B cells are increased in oligoarticular and polyarticular juvenile idiopathic arthritis and their change over time is related to response to tumor necrosis factor inhibitors. Arthritis Rheumatol 2018;70:606-15.

21. Magni-Manzoni S. Ultrasound in juvenile idiopathic arthritis. Pediatr Rheumatol Online J 2016;14:33.

22. Magni-Manzoni S, Scire CA, Ravelli A, Klersy C, Rossi S, Muratore $\mathrm{V}$, et al. Ultrasound-detected synovial abnormalities are frequent in clinically inactive juvenile idiopathic arthritis, but do not predict a flare of synovitis. Ann Rheum Dis 2013;72:223-8. 